\title{
Rapid Eye Movement Sleep Phase Duration
}

National Cancer Institute

\section{Source}

National Cancer Institute. Rapid Eye Movement Sleep Phase Duration. NCI Thesaurus.

Code C154871.

The total amount of time an individual spends in rapid eye movement (REM) sleep. 